\title{
Geographie der (Un-) Sicherheit - Einführung zum Themenheft
}

\author{
Benedikt Korf, Zürich, Jürgen Ossenbrügge, \\ Hamburg
}

Am 29. November 2009 erhielt die Initiative gegen einen Neubau von Minaretten in der Schweiz bei einer Volksabstimmung 57.5\% der abgegebenen Stimmen. Im Vorfeld warben die Initianten für die Zustimmung zur sogenannten «Antiminarettinitiative» mit einem Plakat, das eine Frau in Burka neben einer Phalanx von schwarzen Minaretten zeigt, die auf einer Schweizer Flagge platziert sind und eher aussehen wie Raketen (Abb. 1). Der fundamentalistische Islam, so suggeriert dieses Bild, dringt in die Schweiz ein und stellt eine gravierende Bedrohung dar. Migration, Integration und Religion werden dadurch $\mathrm{zu}$ einem Sicherheitsproblem. Dementsprechend lässt sich das Plakat als bildlicher Ausdruck einer Versicherheitlichung - als englischer Fachterminus securitization - der Migrationspolitik sehen und interpretieren. Doch blieb dieses Plakat, das in der Schweiz und international auf grosse Resonanz stiess, nicht unbeantwortet. «Der Himmel über der Schweiz ist gross genug» plakatierte die Gesellschaft «Minderheiten in der Schweiz» (Abb. 2).
Dieser Vorgang zeigt die Dynamik von Versicherheitlichung auf: Bestimmte politische Fragen werden von Akteuren als Sicherheitsproblem definiert, doch bleiben diese Diskurse und Sprechakte im öffentlichen Raum umstritten, werden herausgefordert und hinterfragt - ein Schritt der desecuritization, der den politischen Raum wieder öffnet (WÆVER 1995).

Versicherheitlichung bezeichnet den gesellschaftlichen Prozess, in dem spezifische Probleme im politischen und öffentlichen Diskurs zu einem Sicherheitsproblem und zu einer gravierenden und manchmal gar existentiellen Bedrohung transformiert werden (BuzAN et al. 1998). Aus der Sicht der kritischen Sicherheitsstudien (critical security studies) sind sicherheitspolitische Themen und Prozesse also sozial konstruierte Phänomene. Derartige Vorgänge einer securitization finden sich in vielen derzeitigen gesellschaftlichen Debatten wieder: Krieg gegen den Terror, Klimakriege, Gesundheitsepidemien, Gewalt in Megastädten, globale Migrationsströme. Analysegegenstände sind Sprechakte und weitergefasste diskursive Praktiken, die nicht nur Ansprachen von Politikern, sondern auch jegliche andere Form von Meinungskundgebung - Einschät-

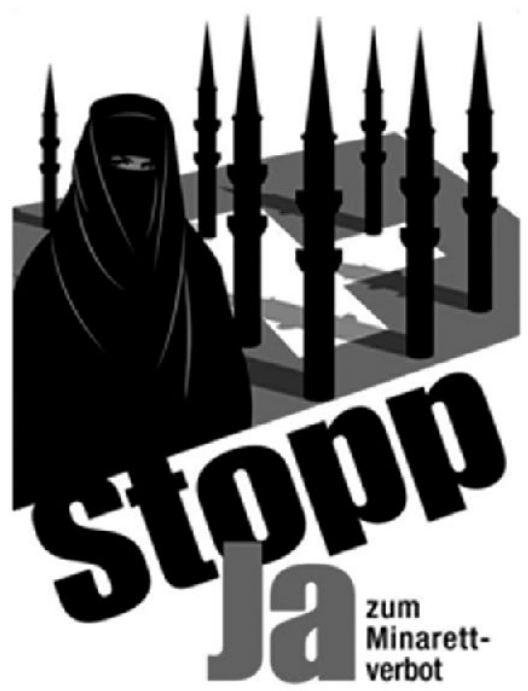

Abb. 1: Das umstrittene Plakat der sogenannten Antiminarettinitiative

The controversial poster of the "Anti-minaret initiative»

L'affiche controversée de l'initiative anti-minarets

Quelle: http:/www.minarette.ch

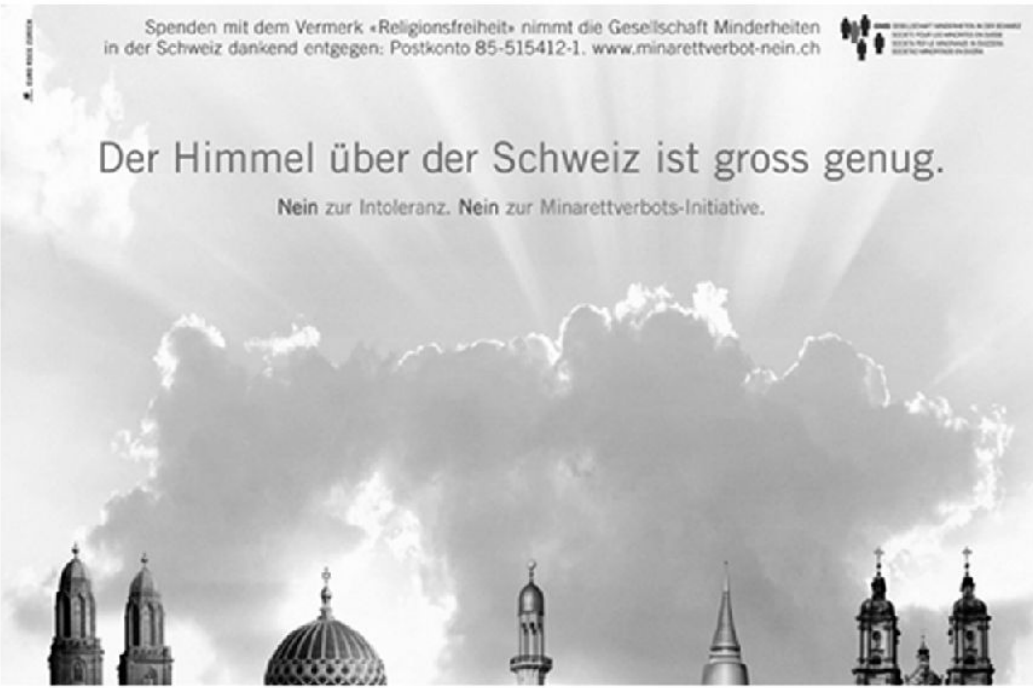

Abb. 2: Ein Plakat als Antwort auf das Plakat der Antiminarettinitiative

A poster response to the "Anti-minaret initiative» poster Une affiche en réponse à l'affiche de l'initiative anti-minarets

Quelle: http:/www.gms-minderheiten.ch/minarettverbot/ informations.php 
zungen von Zeitungen, Beamten und Think Tanks - miteinschließen. Daher lässt sich der Prozess der Versicherheitlichung auch als proaktive Festlegung öffentlicher Gefahren, als Festschreibung von diffusen und unklaren Gefährdungslagen in konkrete Bedrohungen beschreiben. Dieser Vorgang ist hochpolitisch, interessegeladen und machtgetränkt. Der Verweis auf Bedrohung und Gefährlichkeit ermöglicht weiterhin eine Legitimation aussergewöhnlicher, z.T. auch ausserrechtlicher Formen des Regierens: Phänomene, die securitized sind, erfordern aussergewöhnliche Massnahmen, den Ausnahmezustand, die Aussetzung «normaler» Gesetze, die zwar das Individuum schützen, doch gerade damit das Kollektiv bedrohen.

In der erweiterten securitization Debatte, also in den Diskussionen um die soziale Konstruktion und politische Nützlichkeit von öffentlichen Gefahren, kann man verschiedene Denkrichtungen unterscheiden, die grob in drei Schulen eingeordnet werden (vgl. HAGMANN 2010, in diesem Heft): Die «Waliser» Schule (Ken Booth u.a.) problematisiert die Frage, wer von den Diskursen und Praktiken der Sicherheitspolitik betroffen ist: werden Individuen, die «Zivilgesellschaft» oder der Staat zum Zentrum sicherheitspolitischen Handelns erklärt? Die «Pariser» Schule (DIDIER Bigo u.a.) konzentriert sich auf die Gouvernementalität neuer Gefahrendiskurse. Die «Kopenhagener» Schule (Ole WæVER u.a.) zeichnet die Ausgestaltung internationaler Politik mit Freund-Feind-Bildern und die Errichtung extra-legaler Notstandsräume in einzelnen Ländern nach. Innerhalb dieser verschiedenen Denkrichtungen muss noch zwischen einer engen und einer weiten Auslegung des Sicherheitsbegriffs unterschieden werden (vgl. Krause \& Williams 1996). Der enge Sicherheitsbegriff orientiert sich an einem klassischen, nationalstaatlich orientierten Verständnis von Sicherheit und trifft zum Beispiel auf securitization im Rahmen des Krieges gegen den Terror zu, ein erweiterter Sicherheitsbegriff nimmt auch internationale Migrationsströme, Klimakriege, Umweltsicherheit, Gesundheitsrisiken und Gewalt in Megastädten als Sicherheitsproblem wahr.

Erstaunlicherweise hat die Versicherheitlichungsdebatte, die in den Politikwissenschaften breit rezipiert und debattiert wird (vgl. Balzace 2005; Buzan et al. 1998; McDonald 2007; Neocleous 2008; Stritzel 2007; WæVER 1995), bislang wenig Resonanz in der (deutschsprachigen) Politischen Geographie gefunden, obwohl sich vielfältige Überschneidungen mit den critical geopolitics identifizieren lassen (vgl. HAGMANN 2010, in diesem Heft). Beiden Ansätzen geht es um die Identifizierung, oft auch Entlarvung, spezifischer diskursiver Rahmungen und geopolitischer Leitbilder (vgl. DodDS 2007; Gregory 2004; LossaU 2000; Ó Tuathail \& Dalby 1998; Reuber \& Wolkers-
DORFER 2003). Gemeinsam ist critical geopolitics und securitization studies ein gewisser Fokus auf Elitendiskurse, obwohl dies auch zum Gegenstand immanenter Kritik wurde (Müller \& ReubER 2008). Den critical geopolitics ging es dabei vor allem um die räumlichen Semantiken und imaginativen Geographien dieser Leitbilder (REDEPENNING 2006). Durch ihren Fokus auf konstruktivistische Ansätze, eine situative und diskursive Beobachtung der Sicherheitspolitik und die Analyse von Diskursen und sozialer Praxis ist die securitization Debatte an die critical geopolitics anschlussfähig und geht gleichzeitig über diese hinaus (DALBY 2009; INGRAM \& DodDs 2009), u.a. durch die Öffnung zu einem erweiterten Sicherheitsbegriff und damit neuen Themenfeldern. Gleichzeitig bietet die Politische Geographie das Potential, die räumlichen Semantiken und territorialen Praktiken von securitization expliziter in die Analyse einzubinden und damit neue Impulse in die politikwissenschaftliche Debatte zu tragen.

\section{Zielsetzung dieses Themenheftes}

Dieses Themenheft ist ein erster Beitrag, die securitization Debatte innerhalb der (Politischen) Geographie (aber auch: Stadtgeographie, Geographische Entwicklungsforschung, Politische Ökologie) zu positionieren. Die Zusammenstellung der insgesamt sechs Beiträge deckt dabei ein breites Themenspektrum der Versicherheitlichung ab: Der Beitrag von Jonas Hagmann skizziert die Diskurslandschaft der securitization Debatte aus der Sicht der critical security studies und stellt erste Bezüge zu den critical geopolitics her. Die Beiträge von Conrad Schetter und Bernd Belina beziehen sich auf den klassischen Sicherheitsbegriff und behandeln Fragen von «ungoverned territories» im Krieg gegen den Terror (SCHETTER) und die EU-Aussengrenze als Sicherheitsproblem (BELINA). Hier zeigt sich bereits die Wichtigkeit räumlicher Semantiken und territorialer Praktiken als spezifischer Beitrag der Politischen Geographie zur securitization Debatte. Der Beitrag von Johannes Herbeck und Michael Flitner thematisiert den Klimawandel als Bedrohungsdiskurs und diskutiert Implikationen eines erweiterten Sicherheitsbegriffs, wie er in dem Konzept der «human security» zum Ausdruck kommt. Die letzten beiden Beiträge von Marc Redepenning, Henriette Neef und Edvânia Torres Aguiar Gomes sowie Mélina Germes und GEorg GLasze behandeln das Thema der Unsicherheit (in) der Stadt anhand der politischen Regulation von Straßenhändlern in brasilianischen Metropolen und den politischen Debatten um die Bedrohungen und Gefahren der Pariser banlieues.

Damit zeigen diese Beiträge die Bandbreite von Geographien der (Un-) Sicherheit auf und verbinden diese 
mit der Versicherheitlichung politischer Phänomene in verschiedenen gesellschaftspolitischen Diskursräumen. Gleichzeitig verorten die thematischen Beiträge diese Versicherheitlichung in konkreten räumlichen Semantiken und territorialen Alltagspraktiken und verdeutlichen damit den besonderen Beitrag, den die Politische Geographie zur securitization Debatte beisteuern kann. ScHETter zeigt zum Beispiel, wie sich durch den Terminus ungoverned territories das Problem des internationalen Terrorismus wieder territorial - in sogenannten failed states - verorten und mit konventionellen Formen der Kriegsführung (in Afghanistan und anderswo) bekämpfen lässt. GERMEs und GLasze veranschaulichen, wie die banlieues $\mathrm{zu}$ Gegenorten der République werden, indem sie als bedrohliche und fremde Orte konstituiert werden. Bei der jüngsten Fussballweltmeisterschaft gipfelte dieser Diskurs der Gegenorte in der Äusserung des Philosophen Alain Finckielkraut, der die Ungezogenheit und Revolte der französischen Nationalspieler, die sich gegen die Abstrafung eines ihrer Kollegen durch Nationaltrainer RAYMOND DOMENECH aufgelehnt hatten, als Sieg der Unkultur der Vorstädte über die städtische Zivilisation des Landes markierte (RITTE 2010). Für REDEPENNING et al. ist dieser Versuch der Markierung von Gegenorten mittels der raumbezogenen Technik einer Containerisierung jedoch zum Scheitern verurteilt: der öffentliche Raum stellt sich unter aktuellen gesellschaftlichen Raumverhältnissen mehr und mehr als flüssiger Raum dar, Sicherheit und Unsicherheit können nicht mehr territorial abgegrenzt werden und sind somit neu zu denken.

\section{Zur Geographie der (Un-) Sicherheit}

Somit kann Versicherheitlichung nicht ohne die in ihr inhärenten räumlichen Semantiken gedacht werden, bei denen es um das Ein-, Ab- und Ausgrenzen von Objekten und Subjekten geht, aber auch um den Geltungsbereich und die Wirkungsräume von Regeln, Gesetzen und Normen. Darüber hinaus enthält Versicherheitlichung auch eine spezifische Zeitgeographie: Die Bedrohung liegt in der Zukunft, doch macht diese Zukunft, die es vielleicht gar nicht geben wird, $\mathrm{da}$ es sich lediglich um eine potentielle handelt, konkretes Handeln in der Jetztzeit erforderlich: durch Massnahmen der Vorsorge und der Vorsicht (ANDERSoN 2010). (Streng genommen wird in der Literatur zwischen Versicherheitlichung und Risikologik unterschieden: Sicherheit bezieht sich auf Aktualität, Risiko auf Potentiale und präventives Handeln. Wir haben in diesem Themenheft diese Unterscheidung nicht weiter thematisiert; vgl. dazu AARADAU \& VAN MüNSTER 2007). Indem Versicherheitlichung ausserordentliche Massnahmen zur Bannung existentieller Gefahren einfordert, legitimiert sie tendenziell eine gouvernementale Praxis der rechtlichen Ausnahme, der polizeilichen Präventions tat (vgl. OpITz 2008: 217). Es ist die Temporalität des existentiellen Ernstfalls, die die Ausnahme über das bestehende Recht hebt. So ist empirisch von Interesse, wie die überhitzte Rhetorik von securitization mit der taktischen Auslegung oder Suspendierung rechtlicher Regeln einhergeht.

Hierbei gilt es, zwei Formen von Ausnahmezuständen mit eigenen Temporalitäten und verschiedenen Graden von Verrechtlichung oder Domestizierung zu differenzieren. Auf der einen Seite gibt es die von FouCAult angesprochenen permanenten, «kleinen» Ausnahmezustände (Foucault 2004). Diese «kleinen» Ausnahmezustände zeigen sich zum Beispiel in der Praxis von Sicherheitskontrollen an Flughäfen, in der zunehmenden Überwachung öffentlicher Räume und in der Versicherheitlichung der Migrationspolitik in Form strengerer Grenzkontrollen (vgl. BeLiNA 2010, in diesem Heft). Foucault interessiert sich für den Normalzustand, der unterhalb, neben und teilweise auch gegen rechtliche Mechanismen Bestand hat - in Form der «kleinen» Ausnahmezustände. Foucault zielt somit darauf ab, wie «kleine» Ausnahmezustände in den Grauzonen der Legalität Normalität produzieren (vgl. LeMKe 2004). Dem steht ein stärker dezisionistisches Denken des Ausnahmezustands gegenüber. Dieses ist geprägt von CARL ScHMITTs einflussreichem Satz, dass «souverän ist, wer über den Ausnahmezustand entscheidet» ([1922] 2004: 13). Dem deutschen Staatsrechtler Otto DePenheuer geht es zum Beispiel um die Behandlung existentieller Unsicherheit im Augenblick ihres Ereignisses (DEPENHEUER 2007). DePENHEuers Fall ist das entführte Flugzeug, das den existentiellen, grossen Ausnahmezustand hervorruft, den Ernstfall, der eine Entscheidung zum sofortigen Handeln notwendig macht, zu einem Handeln, das auch über das Recht hinausgehen kann - und damit den handelnden Staat als Souverän begründet in der Entscheidung (Dezision).

In der Praxis zeigt sich aber das Ineinandergreifen von «kleinen» Ausnahmezuständen und existentiellem (oft nur gedachten, antizipierten) grossen Ausnahmezustand, der in die Jetztzeit projiziert wird. Diese politische Geographie des Rechtlichen, die im Prozess der Versicherheitlichung entsteht, bezeichnet AGAMBEN als Diffusion der Ausnahme in die Normalität (AgAmben 2004). Der einer erfolgreichen Versicherheitlichung folgende Ausnahmezustand führt zu einer Entgrenzung der Rechtslogik. Aus dieser ergeben sich Möglichkeiten zur Durchsetzung neuer Rechtsvorstellungen. Ein Recht jedoch, das Ausnahmezustände «verrechtlicht», verändert sich (auch im normativen Sinne) und transformiert damit die demokratische Verfasstheit eines Gemeinwesens und die institutionellen Rahmenbedingungen gesellschaftlicher Teilhabe. 
Auch die Raketenminarette der Schweizer Antiminarettinitiative sind letztlich Ausdruck einer Geographie von (Un-) Sicherheit. ULRICH SCHLÜER, einer der Initianten der Antiminarettinitiative, schreibt: «Wo sich Einwanderer der Integration verschliessen, geht es um Eroberung - Eroberung von innen her» (Schlüer 2010). SchlüER konstruiert eine existentielle Bedrohung, die aus dem Phänomen Islam und Migration in der Schweiz ein Problem der (Un-) Sicherheit macht. Getreu der Maxime «Wehret den Anfängen» wurde das Minarett zum Symbol einer drohenden Islamisierung der Schweiz erklärt (obwohl es zum Zeitpunkt der Abstimmung lediglich vier Minarette in der Schweiz gab). Hier zeigt sich - visualisiert im Plakat - eine Geographie der Angst, die das Fremde in «uns», «unserem Land», als existentielle Gefahr zeichnet. Diese Geographie der Angst zieht eine Geographie des Rechts nach sich: das Verbot zum Bau neuer Minarette «auf Schweizer Boden»- als Fremdes, dem Schweizer Rechtswesen und der Demokratie widersprechend. So wird der Akt der Volksabstimmung gleichzeitig zur Wiedereinsetzung der Normalität, zur Rehabilitation des Rechts des Volkes als Souverän - und eine Tat der Vorsorge gegen die Bedrohungen der Zukunft, hier die Angst vor der wachsenden Zahl von Muslimen. Es war also die Angst vor einer antizipierten Gefahr, die die Versicherheitlichung der sogenannten Integrationsfrage von Muslimen in der Schweiz vorangetrieben hat. Damit werden Räume der Unsicherheit sowohl durch Prozesse der Ausgrenzung als auch durch das ins-Präsens-holen postulierter Potentiale geschaffen. Und dennoch: «Der Himmel über der Schweiz ist gross genug», so plakatierte die Gesellschaft «Minderheiten in der Schweiz». Versicherheitlichung bleibt ein im öffentlichen Raum umstrittener Akt.

\section{Literatur}

AARADAU, C. \& R.van Münster (2007): Governing terrorism through risk: taking precautions, un(knowing) the future. - In: European Journal of International Relations 13, 1: 89-115.

Agamben, G. (2004): Ausnahmezustand. - Frankfurt am Main: Suhrkamp Verlag.

Anderson, B. (2010): Preemption, precaution, preparedness: anticipatory action and future geographies. - In: Progress in Human Geography (in press).

BalzacQ, T. (2005): The three faces of securitization: political agenda, audience and context. - In: European Journal of International Relations 11, 2: 171-201.

BelinA, B. (2010): Wie und warum Staat Sicherheit produziert. Dargestellt anhand der Versicherheitlichung grenzüberschreitender Mobilität seitens der EU und der so produzierten Grenzräume. - In: Geographica Helvetica 65, 3: 189-197.

BuZAN, B., WæVER, O. \& J. DE WILdE (1998): Security: a new framework for analysis. - Boulder, London: Lynne Rienner Publishers.

DALBY, S. (2009): Critical geopolitics and security. - In: Burgess, P. (Hrsg.): Handbook of new security studies. - London: Routledge: 50-58.

Depenheuer, O. (2007): Selbstbehauptung des Rechtsstaates. - Paderborn: Schöningh.

DodDs, K. (2007): Geopolitics: a very short introduction. - Oxford, New York: Oxford University Press.

Foucault, M. (2004): Geschichte der Gouvernementalität, Band 1: Sicherheit, Territorium, Bevölkerung. - Vorlesung am Collège de France 1977-1978, Frankfurt am Main: Suhrkamp.

Gregory, D. (2004): The colonial present: Afghanistan, Palestine, Iraq. - Oxford und Malden, MA: Blackwell. Hagmann, J. (2010): Räume der Unsicherheit: Konstruktion, Emanzipation und Exklusion durch Sicherheitspolitik. - In: Geographica Helvetica 65, 3: 172180.

InGRAM, A. \& K. DodDs (Hrsg) (2009): Spaces of security and insecurity. - Aldershot: Ashgate.

Krause, K. \& M.C. Williams (1996): Broadening the agenda of security studies: politics and methods. - In: Mershon International Studies Review 40, 2: 229-254 Lemke, T. (2004): Die Regel der Ausnahme. Giorgio Agamben über Biopolitik und Souveränität. - In: Deutsche Zeitschrift für Philosophie 52, 6: 943-963.

Lossau. J. (2000): Anders denken. Postkolonialismus, Geopolitik und Politische Geographie. - In: Erdkunde 54: $157-168$.

McDonald, M. (2007): Securitization and the construction of security. - In: European Journal of International Relations 14, 4: 563-587.

Müller, M. \& P. Reuber (2008): Empirical verve, conceptual doubts: looking from the outside in at critical geopolitics. - In: Geopolitics 13, 3: 458-472.

Neocleous, M. (2008): Critique of security. - Edinburgh: Edinburgh University Press.

OpITZ, S. (2008): Zwischen Sicherheitsdispositiven und Securitization: Zur Analytik illiberaler Gouvernementalität. - In: Purtschert, P. (Hrsg.): Gouvernementalität und Sicherheit. Zeitdiagnostische Beiträge im Anschluss an Foucault. - Bielefeld: transcript: 201-228. Ó Tuathail, G. \& S. Dalby (1998): Rethinking geopolitics. - London: Routledge.

RedePenning, M. (2006): Wozu Raum? Systemtheorie, critical geopolitics und raumbezogene Semantiken. - = Beiträge zur Regionalen Geographie Europas 62, Leipzig: Leibniz-Institut für Länderkunde.

Reuber, P. \& G. Wolkersdorfer (2003): Geopolitische Leitbilder und die Neuordnung der globalen Machtverhältnisse. - In: Gebhardt, H., Reuber, P. \&. G. WoLkersdorfer (Hrsg.): Kulturgeographie. Aktuelle Ansätze und Entwicklungen. - Heidelberg: Spektrum Verlag: 47-66.

RITTE, J. (2010): Black-Blanc-Out:Frankreich im Schockzustand. - In: Neue Zürcher Zeitung vom 25.6.: 57. 
ScHLÜER, U. (2010): Schweizer Souveränität oder Fremddiktat. Volksinitiative gegen den Bau von Minaretten, Newsletter 1.7.2010; http://www.minarette.ch/ 12.7.2010.

SchmitT, C. ([1922] 2004): Politische Theologie. Vier Kapitel zur Lehre von der Souveränität. - Berlin: Dunckler \& Humblot.

STRITZEL, H. (2007): Towards a theory of securitisation: Copenhagen and beyond. - In: European Journal of International Relations 13, 3:357-383.

WÆVER, O. (1995): Securitization and de-securitization. - In: Lipschutz, R. (Hrsg.): On security. - New York: Columbia University Press: 46-86.

Prof. Dr. Benedikt Korf, Geographisches Institut, Universität Zürich, Winterthurerstrasse 190, CH-8057

Zürich, Schweiz.

e-mail: benedikt.korf@geo.uzh.ch

Prof. Dr. Jürgen Ossenbrügge, Institut für Geographie, Universität Hamburg, Bundesstrasse 55, D-20146 Hamburg, Deutschland.

e-mail: ossenbruegge@geowiss.uni-hamburg.de 